\title{
Keep an eye for all remote complications of caesarean section
}

\author{
Jan Deprest
}

Published online: 25 October 2013

(C) Springer-Verlag Berlin Heidelberg 2013

The most common operation women get is a caesarean section (CS). If the global rate of CS would be around $10 \%$, that means there would be 13 million CS per year or 24 a minute [1]. This operation has its inherent immediate complications, infection being the most common one (17\%). As CS rates increase worldwide, its postoperative complications will only increase. Infection is easy to treat; therefore, it is less feared. Some immediate complications may be life threatening, such as thromboembolic events, for which there is fortunately enough awareness, and most take prophylactic measures.

$\mathrm{CS}$ also has remote complications, and again, these may be life threatening. Obstetricians are very aware of uterine dehiscence (around $1.1 \%)$ or uterine rupture $(0.2 \%$ for elective CS, $0.4-0.6 \%$ for women offered trial of labour) [1]. The most worrying delayed complication is abnormal placentation. The incidence of placenta accreta has risen from 1 in 4,027 deliveries in 1970 to a staggering 1 in 533 deliveries in the previous decade [2]. This increase is likely secondary to the rising rate of caesarean delivery, which in Latin and North America has climbed to over $30 \%[1,3]$. The prenatal diagnosis of placenta accreta, e.g. by ultrasound or Magnetic Resonance, is of utmost importance to aid in delivery planning and improved outcomes.

One less infamous complication is the ectopic presence of functional endometrial tissue in the CS scar [4]. Typically, this will present as an endometrioma causing one or more problems such as cyclical pain, (periodical) swelling and, in some women, pseudo-menstrual blood loss. Endometriosis of the abdominal scar is quite down on the list of endometriosis location sites, which may lead to delayed diagnosis.

The exact incidence of CS scar endometriosis is a matter of debate. In the current issue of Gynecological Surgery,

\section{J. Deprest $(\triangle)$}

Department Development and Regeneration, Organ System Cluster and Obstetrics and Gynaecology, University Hospitals Leuven, Biomedical Sciences, Leuven, Belgium

e-mail: Jan.Deprest@uzleuven.be
Adriaanse et al. report on their attempt to assess its incidence. They expressed the number of surgical diagnoses to the number of caesarean sections done over a given time period, and come up with a $0.95 \%$ incidence. This is more than what was the average of four larger studies previously done [5]. Our Dutch colleagues think this $1 \%$ might still be an underestimation as only symptomatic women who had undergone an operation were included in their study, though neither the available data nor the current design allows a true estimation. Recently, investigators from Sweden performed a prospective cohort study, starting from their birth registry. They, in contrast, observed a $0.1 \%$ risk for developing scar endometriosis, without increased risk following two caesarean deliveries [6]. What is more relevant in that latter study is that CS was associated with an overall increased risk for pelvic endometriosis hazard ratio of 1.8 (95\% CI 1.7-1.9). The risk for endometriosis increased over time: one additional case of endometriosis was found for every 325 women undergoing caesarean section within 10 years. No increase in risk could be seen after two caesarean deliveries.

However, the pathogenesis of post-delivery endometriosis is uncertain. Intuitively, the most logical mechanism is secondary implantation of degenerating decidual cells, which after vaginal delivery may also cause endometriosis in the episiotomy scar. Conversely, abdominal delivery would lead to pelvic or abdominal scar endometriosis. Therefore, it has been advocated to try to prevent spilling of decidua by avoiding contact of swabs, which have previously been used to strip the uterus of remaining placental tissue, with the abdominal scar [7]. Whether this is relevant remains unknown.

In conclusion, a history of caesarean section should increase awareness of both pelvic and scar endometriosis. As to scar endometriosis, though rare, it should be suspected in the presence of a lump causing (cyclical) pain in the abdominal scar. In the Dutch study, one third of diagnoses were made by a surgeon, which may indicate that gynaecologists have overlooked the diagnosis. On average, women were operated on 4 years after their last CS. However, 
most striking is that not any of the women affected had a history of endometriosis, meaning that the awareness of the clinician will only be raised by proper history taking and, obviously, clinical examination-perhaps ideally around the time of maximum complaints. Given that the average implant typically measures over $2 \mathrm{~cm}$, it should be palpable [5]. In spite of this, in another study, larger endometriomas were even longer overlooked than smaller lesions [7]. The condition may be confused with abscess, hematoma, suture granuloma, desmoid tumour, sarcoma and even metastatic malignancy. Once suspected, the lesion can be demonstrated by noninvasive diagnostic tools. The presence of blood products in an anterior abdominal wall mass at magnetic resonance imaging with no other explanation is strongly suggestive of scar endometriosis. Certainty comes only after pathology examination, which may be initially fine needle aspiration, but eventually by wide resection of the endometrioma. The Dutch study confirms resection is effective as no recurrence was demonstrated.

\section{References}

1. CAESAR study collaborative group (2010) Caesarean section surgical techniques: a randomised factorial trial (CAESAR). BJOG 117(11): 1366-1376

2. Wu S, Kocherginsky M, Hibbard JU (2005) Abnormal placentation: twenty-year analysis. Am J Obstet Gynecol 192(5):1458-1461

3. MacDorman MF, Menacker F, Declercq E (2008) Cesarean birth in the United States: epidemiology, trends, and outcomes. Clin Perinatol 35(2):293-307, v

4. Gidwaney R, Badler RL, Yam BL, Hines JJ, Alexeeva V, Donovan V, Katz DS (2012) Endometriosis of abdominal and pelvic wall scars: multimodality imaging findings, pathologic correlation, and radiologic mimics. Radiographics 32(7):2031-2043

5. Adriaanse B, Natté R, Hellebrekers J (2013) Scar endometriosis after a caesarean section: a perhaps underestimated complication. Gynecol Surg doi:10.1007/s10397-013-0807-3

6. Andolf E, Thorsell M, Kallen K (2013) Caesarean section and risk for endometriosis: a prospective cohort study of Swedish registries. BJOG 120(9):1061-1065

7. Francica G (2012) Reliable clinical and sonographic findings in the diagnosis of abdominal wall endometriosis near cesarean section scar. World J Radiol 4(4):135-140 